\title{
Ultrasound findings and histological features of ductal carcinoma in situ detected by ultrasound examination alone
}

\author{
Ayumi Izumori $\cdot$ Koji Takebe $\cdot$ Akira Sato
}

Received: 28 October 2008/Accepted: 14 April 2009/Published online: 3 July 2009

(c) The Japanese Breast Cancer Society 2009

\begin{abstract}
Background With the increasing use of high-resolution ultrasound (US) examination, many breast carcinomas that cannot be identified by mammography (MMG) alone have been detected. Many of these carcinomas are ductal carcinoma in situ (DCIS) and small-sized invasive carcinomas. Until date, DCISs have often been described as palpable masses with calcifications on MMG, but what are the characteristics of DCISs that are detectable by US alone?

Methods One hundred fifty cases with DCIS that we experienced at our clinic from 2003 to 2007 were classified into 47 cases (echo group) diagnosed by US alone and 103 cases (MMG/PE group) diagnosed by MMG or clinically. Results US findings of the echo group showed cystic or solid lesions in 37 cases (79\%). The mean age of the echo group was significantly higher than that of the MMG/PE group (59.6 vs. 51.2 years; $P<0.01$ ). Tumor sizes detected by US were $5.7+2.8$ and $11.5+10.8 \mathrm{~mm}(P<$ 0.001 ), respectively. The tumor sizes of the echo group were, therefore, approximately half that of the MMG/PE group. Extensive intraductal components were significantly fewer in the echo group, and tumor grades of the echo group were significantly low (Van Nuys classification). In the echo group, all cases with a tumor size $<5 \mathrm{~mm}$ were grade 1 by Van Nuys classification. In addition, cases with
\end{abstract}

A. Izumori $(\varangle) \cdot K$. Takebe

Takebe Breast Care Unit, 365-9 Tahishimomachi,

Takamatsu, Kagawa 761-8075, Japan

e-mail: izuiso@mail2.netwave.or.jp

A. Sato

Takamatsu Heiwa Hospital, 1-4-1 Ritsurincho,

Takamatsu, Kagawa 760-0073, Japan $\geq 5 \mathrm{~mm}$ tumor size had a significantly lower tumor grade in the echo group than in the MMG/PE group.

Conclusions Cystic or solid lesions accounted for approximately $80 \%$ of US findings of DCISs detected by US alone, and most were similar to benign forms. Moreover, most DCISs detected by US alone were localized and of low grade (Van Nuys classification).

Keywords Ductal carcinoma in situ (DCIS) . Noninvasive ductal carcinoma $\cdot$ Ultrasound . Ultrasonography · Van Nuys classification

\section{Introduction}

The introduction of mammography (MMG) as an adjunct in breast screening examination has increased the detection frequency of ductal carcinoma in situ (DCIS). At the same time, breast ultrasonography (US) examination in women with dense breast tissue has led to the detection of breast cancers that were not identified on MMG. The frequency of breast cancses detected by US is approximately $0.3-0.4 \%$. US has been frequently reported to be more sensitive than MMG [1-3], and many DCISs have been detected by US. However, currently there is no definite opinion on US findings of DCISs, which have been reported as irregular masses, mammary duct ectasia [2, 4, 5], and benign cystoids $[1,5]$. Furthermore, in these reports, many cases that are detectable by MMG were included. We performed US in all outpatients that visited our clinic, and those with positive findings underwent detailed examination mainly via aspiration biopsy cytology. As a result, we could detect many DCISs by US alone [6]. In this study, we summarize the features of DCISs detected by US alone based on US and clinicopathological findings. 


\section{Patients and methods}

From January 2003 to December 2007, we examined 35,886 women. Of those, 2,812 masses were sampled by ultrasound-guided fine-needle aspiration cytology. We found 729 cases of primary breast cancers at our clinic, of which 150 cases $(20.6 \%)$ diagnosed with pathological DCISs were included in this study. They were retrospectively classified by one author into cases detected by US alone (echo group) and cases detected by MMG or clinical findings, such as palpable mass and abnormal nipple discharge (MMG/PE group), and examined clinicopathologically. The following US devices were used: ALOKASSD1000, ALOKA-SSD5000, TOSHIBA XARIO, and APRIO. In all cases, MMG and US were performed regardless of age. Cases with shape irregularities, internal echo irregularities, segmental and solitary cystic lesions in the elderly, etc., on US examination were examined in detail [6] regardless of tumor size. For cases that required detailed examination and those in which lesions were observed by US, aspiration biopsy cytology was performed using a $23 \mathrm{G}$ needle. When MMG revealed calcifications and the lesions could not be determined by US, mammotome biopsy was performed. For pathological investigation, the resected specimens were cleaved at 2-5-mm intervals, and sections were prepared. For classification of DCIS histological subtypes, comedo necrosis of $\geq 50 \%$ of the gross tumor was categorized as comedo type and the others as non-comedo type. For the assessment of extensive intraductal components, those located $\geq 2 \mathrm{~cm}$ from the periphery of the main lesions recognized by US were defined as positive. For the pathological classification of DCIS, Van-Nuys classification [7] was used. The US and clinicopathological findings were statistically analyzed by $\chi^{2}$ analysis and $t$ test.

\section{Results}

\section{US findings of DCIS cases}

Based on US findings, DCIS was classified into cystic or solid mass (56 cases, Fig. 1), ill-defined hypoechoic mass (34 cases, Fig. 2), microlobulated mass (22 cases, Fig. 3), duct dilatation (7 cases, Fig. 4), and calcification (7 cases). DCIS features were classified as follows: cystic or solid mass (hypoechoic or solid mass with clear margins, spherical in shape or up to 4 scant nicks), ill-defined hypoechoic mass (horned), microlobulated mass (mammary duct appears dilated, inner structure with a hypoechoic pattern), duct dilatation (one mammary duct is dilated), calcification (presence of echogenic spots that appear to be calcifications; however, background changes of the mammary gland are not helpful).

\section{US findings in echo and MMG/PE groups}

Echo and MMG/PE groups consisted of 47 and 103 cases, respectively (Table 1). In the latter, 23 patients could not be diagnosed by US. Thirty-seven patients (79\%) of the echo group had a cystic or solid mass, whereas in the MMG/PE group, cystic or solid mass, illdefined hypoechoic mass, microlobulated mass, and duct dilatation comprised $18,25,20$, and $6 \%$, respectively $(P<0.001)$.

Characteristics of mass-forming types identified by US in echo and MMG/PE groups

For cases with a cystic or solid and ill-defined hypoechoic masses, the shape, margin, echogenicity, echogeneity, and
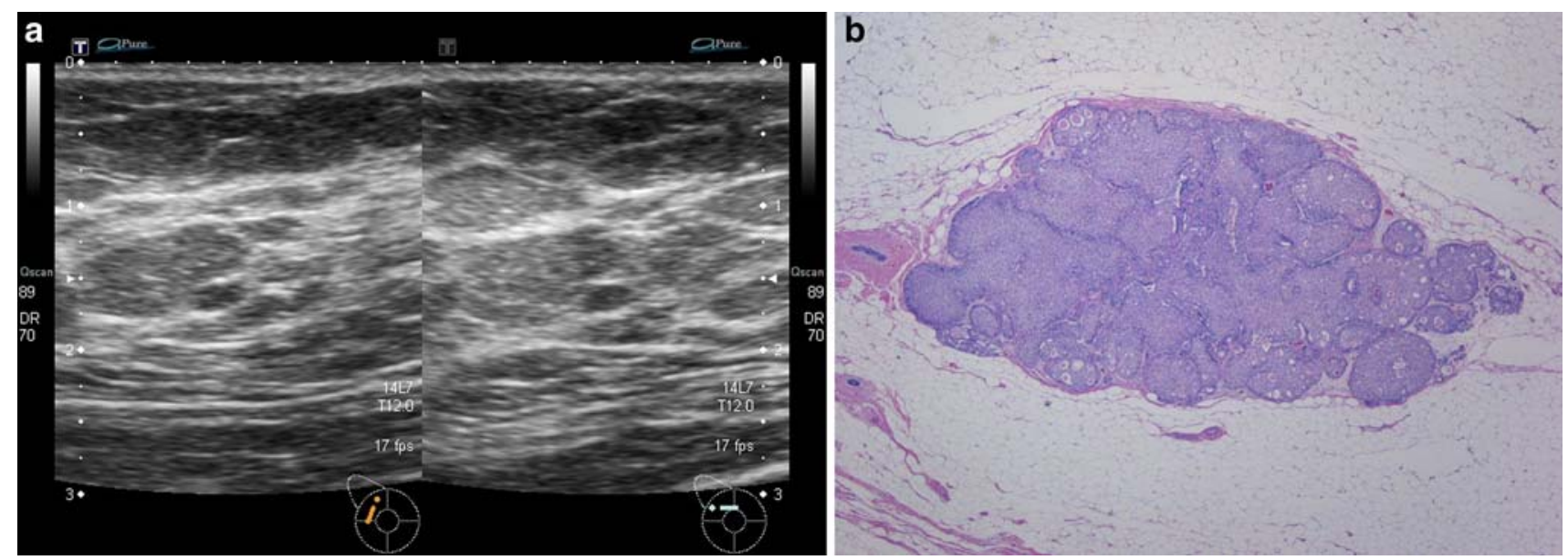

Fig. 1 a Cystic or solid mass; b NG1 

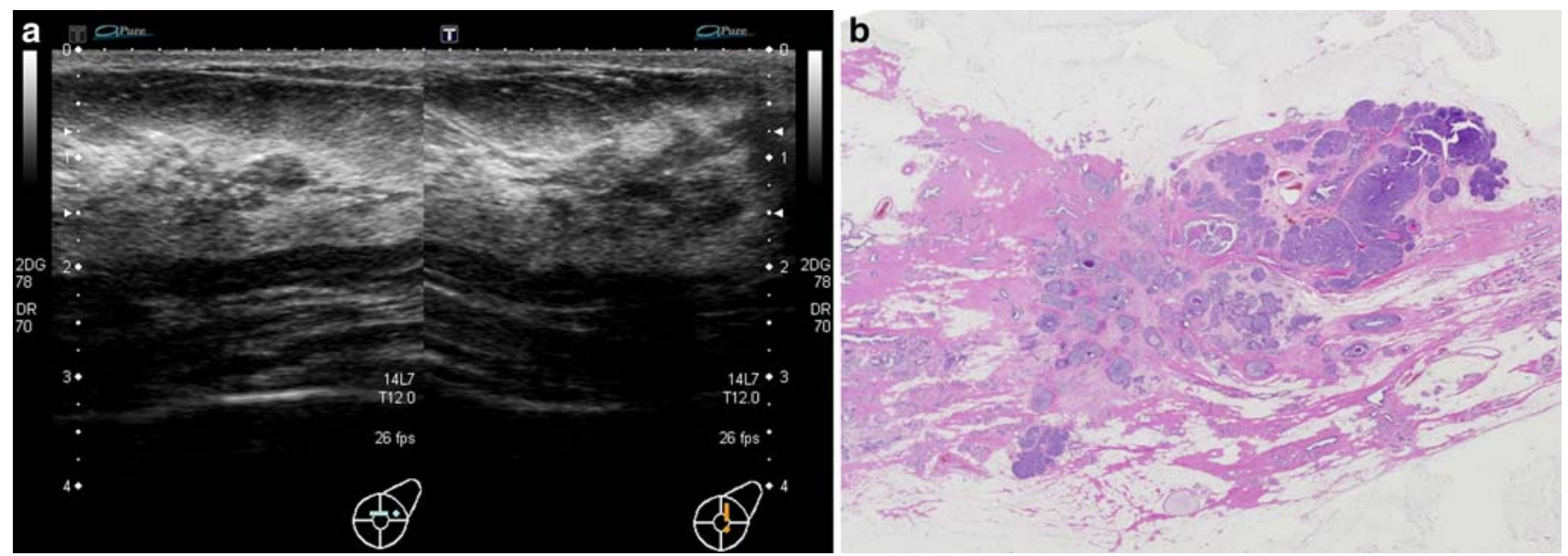

Fig. 2 a Ill-defined hypoechoic mass; b NG2
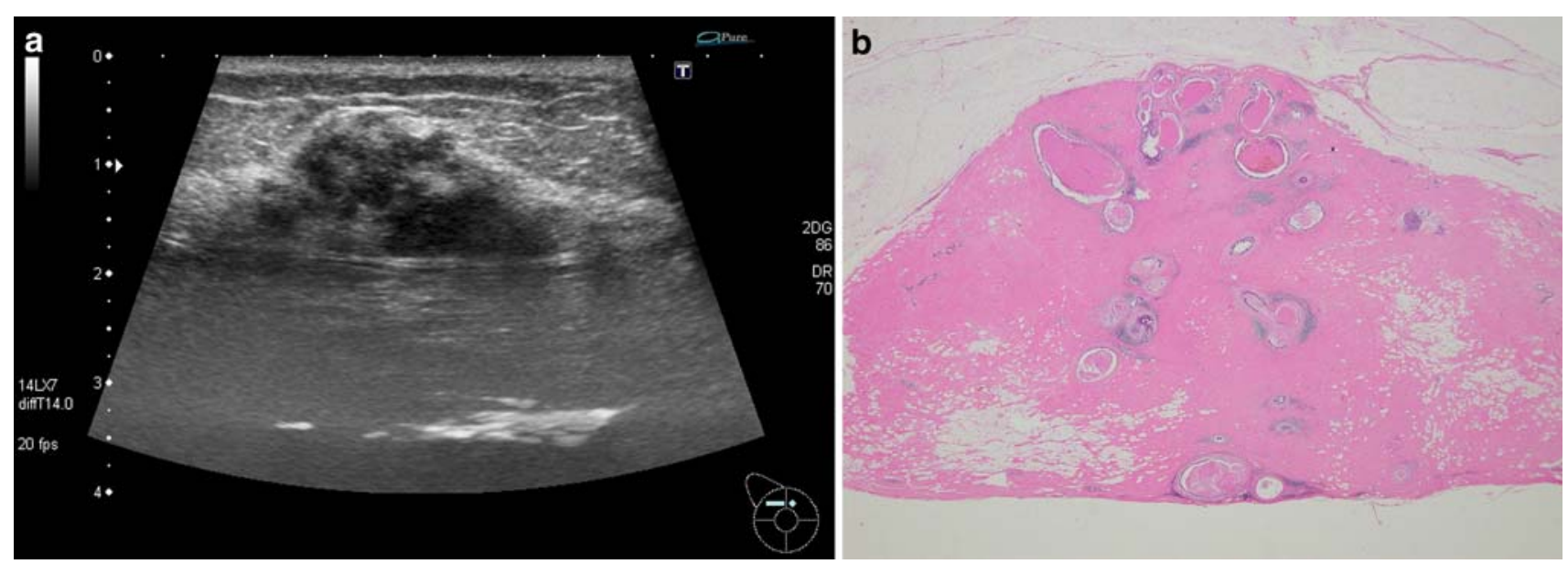

Fig. 3 a Microlobulated mass; b NG3
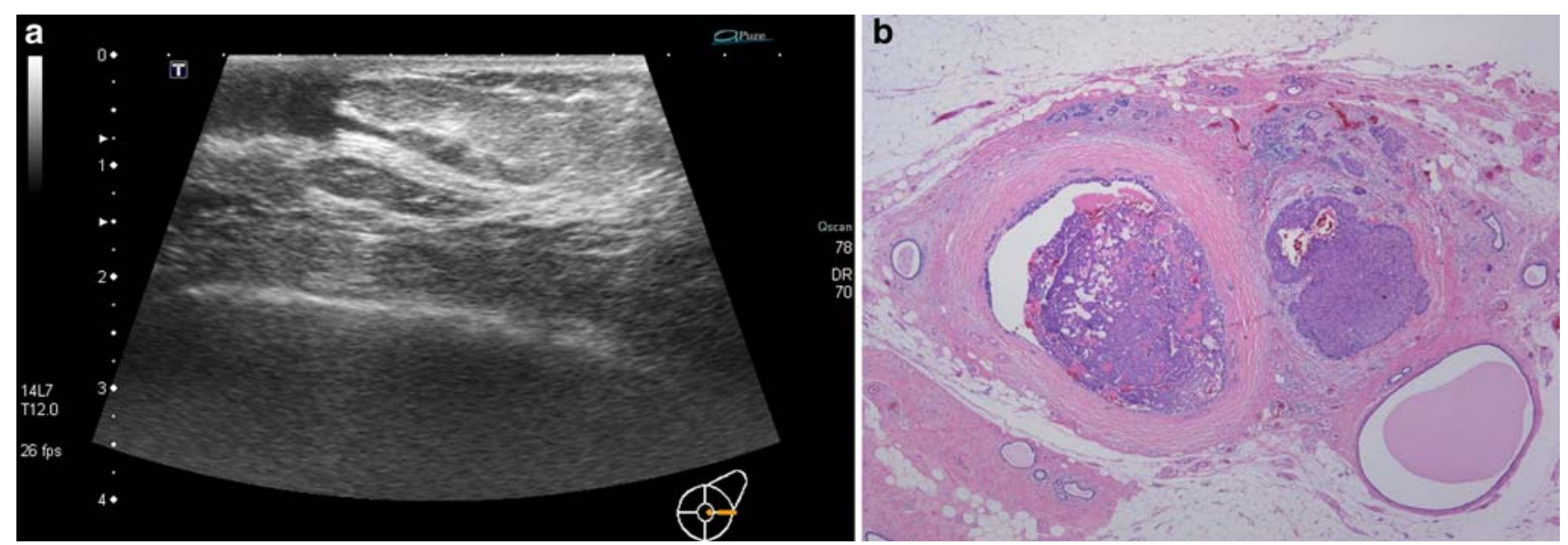

Fig. 4 a Duct dilatation; b NG1

posterior phenomena were examined (Table 2). As a result, ovoid and irregular shapes were frequently observed in the echo and MMG/PE groups $(P<0.001)$, respectively.
While margins in the echo group were circumscribed (42\%) and microlobulated (49\%), those in the MMG/PE group tended to be microlobulated and indistinct 
Table 1 Ultrasound Findings of DCIS in echo group versus MMG/ PE group

$\left.\begin{array}{lcc}\hline \text { Ultrasound Findings } & \text { Echo -group }(\mathrm{n}=47) & \text { MMG/PE-group }(\mathrm{n}=103) \\ \hline \text { Cystic or solid mass } & 37 & 17 \\ \text { Ill-definded hypoechoic mass } & 8 & 28 \\ \text { Microlobulated mass } & 1 & 22 \\ \text { Duct dilatation lesion } & 1 & 6 \\ \text { Calcification lesion } & 0 & 7 \\ \text { not visuable } & 0 & 23\end{array}\right]^{*}$

$* P<0.001$

Table 2 Ultrasound features of cystic or solid masses and ill-defined hypoechoic masses in the echo group versus MMG/PE group ultrasound features

\begin{tabular}{|c|c|c|}
\hline & Echo-group $(\mathrm{n}=45)$ & MMG/PE-group $(n=45)$ \\
\hline \multicolumn{3}{|l|}{ Shape } \\
\hline Ovoid & 27 & $107^{*}$ \\
\hline Lobular & 12 & 12 \\
\hline Irregular & 6 & $23-$ \\
\hline \multicolumn{3}{|l|}{ Margin } \\
\hline Circumscribed & 19 & $127^{* *}$ \\
\hline Microlobulated & 22 & 15 \\
\hline Indistinct & 4 & $18-$ \\
\hline \multicolumn{3}{|l|}{ Echogenicity } \\
\hline Hypoechoic & 24 & $297^{* * * *}$ \\
\hline Isoechoic & 21 & $16-$ \\
\hline \multicolumn{3}{|l|}{ Echogeneity } \\
\hline Heterogeneous & 22 & $317^{* * *}$ \\
\hline Homogeneous & 23 & $14-$ \\
\hline \multicolumn{3}{|l|}{ Posterior phenomena } \\
\hline Nil & 41 & 42 \\
\hline Enhanced & 4 & 3 \\
\hline
\end{tabular}

$* P<0.001, * * P<0.01$, *** $P<0.1$

$(P<0.01)$. Homogeneous echogeneity was observed in half of the cases of the echo group, while in $69 \%$ of the MMG/PE group, heterogeneous echogeneity was observed. In both groups, there were almost no cases with posterior phenomena.

Clinicopathological examination in echo (47 cases) and MMG/PE (103 cases) groups

Mean age of the echo and MMG/PE groups was 59.6 and 51.2 years, respectively; it was significantly higher in the echo group $(P<0.01)$. In the MMG/PE group, 40 patients $(38.8 \%)$ had symptoms such as awareness of a lump and bloody nipple discharge. On MMG, 87 cases $(84.5 \%)$ were of grade $\mathrm{C} 3$ or higher, and on US, similar findings were seen in 88 cases $(85.4 \%)$. Mean mass diameters of the echo and MMG/PE groups were $5.7 \pm 2.8$ and $11.5 \pm 10.8 \mathrm{~mm}$ $(P<0.001)$, respectively, i.e., the mean mass diameters of the echo group were approximately half that of the MMG/ $\mathrm{PE}$ group. Furthermore, mass diameters of the MMG/PE group varied in size. Extensive intraductal components were observed in 19 (40.4\%) and $82(79.6 \%)$ cases, respectively, and were significantly less in the echo group. While in the MMG/PE group, comedocarcinomas were observed in 7 of 103 cases, in the echo group, they were observed only in 1 of 47 cases. Only one case in each group had grades 2 and 3 (Van Nuys classification), and tumor grades were significantly lower than in the MMG/PE group. Additionally, in comparison cases detected by MMG alone (MMG group), extensive intraductal componets were significantly fewer in the echo group, and tumor grades of the echo group were significantly low. The tumor size of the MMG group was ductal spread (Table 3).

Clinicopathological findings classified based on US tumor size

US tumor size was classified into $<5$ and $\geq 5 \mathrm{~mm}$ groups, and pathological findings were examined (Table 4). In the echo group, $<5 \mathrm{~mm}$ tumors were observed in 29 cases $(61.7 \%)$ and $\geq 5 \mathrm{~mm}$ in 18 cases $(38.3 \%)$. In the MMG/PE group, $<5 \mathrm{~mm}$ tumors were observed in 16 cases $(15.7 \%)$ and $\geq 5 \mathrm{~mm}$ in 64 cases $(62.1 \%)$. An overall tumor size of $<5 \mathrm{~mm}$ were observed in 45 cases (30\%). Tumor size was not associated with the presence of extensive intraductal components in both echo and MMG/PE groups. In the echo group, all cases with tumor sizes $<5 \mathrm{~mm}$ were grade 1 (Van Nuys classification), indicating low tumor grades. Moreover, in the MMG/PE group, cases with tumor sizes $<5 \mathrm{~mm}$ had significantly lower tumor grades than those with tumor sizes $\geq 5 \mathrm{~mm}$. In the echo group, in cases with

Table 3 Clinico-pathological findings of DCIS

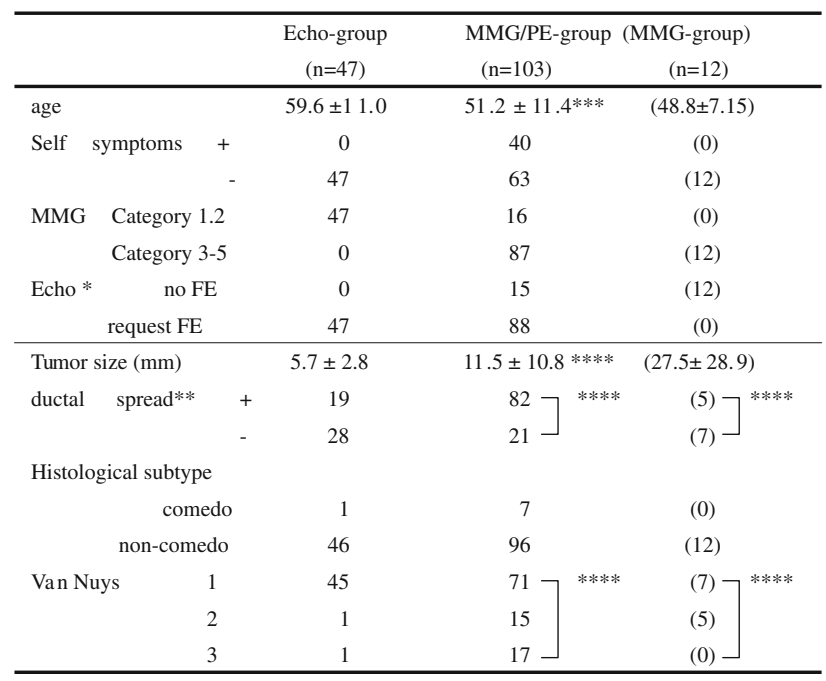

FE Further examination

* Authors' classification; ** + : cases with ductal spread $\geq 2 \mathrm{~cm}$; $* * * P<0.01 ; * * * * P<0.001$ 
Table 4 Clinico-pathological findings of DCIS between tumor size(US)

\begin{tabular}{|c|c|c|c|c|c|}
\hline & & \multicolumn{2}{|c|}{ Echo-group } & \multicolumn{2}{|c|}{ MMG/PE -group } \\
\hline \multicolumn{2}{|l|}{ Tumor Size(US) } & $\begin{array}{l}<5 \mathrm{~mm} \\
(\mathrm{n}=29)\end{array}$ & $\begin{array}{l}\geqq 5 \mathrm{~mm} \\
(\mathrm{n}=18)\end{array}$ & $\begin{array}{l}<5 \mathrm{~mm} \\
(\mathrm{n}=16)\end{array}$ & $\begin{array}{l}\geqq 5 \mathrm{~mm} \\
(\mathrm{n}=64)\end{array}$ \\
\hline \multicolumn{2}{|l|}{ Age } & $61 \pm 10.4$ & $49.9 \pm 8.5$ & $49 \pm 10.6$ & $52.5 \pm 11.8 *$ \\
\hline \multicolumn{2}{|l|}{ Tumor size(mm) } & $3.9 \pm 1.0$ & $8.3 \pm 3.5$ & $4.0 \pm 0.9$ & $15.1 \pm 13.4$ \\
\hline \multirow[t]{2}{*}{ ductal spread } & + & 10 & 9 & 11 & 50 \\
\hline & - & 20 & 8 & 5 & 14 \\
\hline \multicolumn{6}{|l|}{ Histological } \\
\hline \multirow{2}{*}{$\begin{array}{l}\text { subtype } \\
\text { nor }\end{array}$} & medo & 0 & 1 & 0 & 7 \\
\hline & omedo & 29 & 17 & 16 & 57 \\
\hline \multirow[t]{3}{*}{ Van Nuys } & 1 & 29 & 16 & 12 & $417^{* *}$ \\
\hline & 2 & 0 & 1 & 3 & 7 \\
\hline & 3 & 0 & 1 & 1 & $16-$ \\
\hline
\end{tabular}

$* P<0.005, * * P<0.05$

tumor sizes $\geq 5 \mathrm{~mm}$, tumor grades were lower than those in the MMG/PE group.

\section{Discussion}

Through a wider use of MMG, the frequency of DCIS detection is increasing [9-13]. While many DCISs are detected through microcalcifications [11-13], those without calcifications cannot be detected by MMG, and 6-23\% DCIS are said to remain undetected [5, 11-15]. At the same time, by breast US, smaller breast carcinomas can be detected than by MMG alone, and a high breast carcinoma detection rate $(0.31-0.4 \%)$ has been reported in which the frequency of DCISs was 11-14\% [1-3]. US, in particular, is believed to be useful for detecting breast carcinomas in young people with dense breast tissue [1-3]. However, as most DCISs detected by US that have been reported so far were comedo type, many of them were also observed as abnormal by MMG and frequently accompanied by subjective symptoms $[6$, $12-14,16]$. We performed US in all outpatients that visited our clinic, and those with positive findings underwent detailed examination. As a result, many DCISs without subjective symptoms could be detected by US alone [7].

In this study, we examined 150 cases with DCIS using US and retrospectively classified them into a cystic or solid mass, ill-defined hypoechoic mass, microlobulated mass, duct dilatation, and calcification. Among these, in 37 (79\%) of 47 cases with US findings alone, a cystic or solid mass was observed. Most were ovoid in shape, and the margins were circumscribed or microlobulated, making it difficult to differentiate from benign lesions. Moreover, approximately half of these cases had heterogeneous internal echoes.
Moon et al. [6] reported DCISs detected by US; however, they performed US in subjects with dense breast tissue and subjective symptoms, and almost all cases had calcifications. Thus, this study cannot be compared with ours. Chen et al. [16] reported that noncomedo type DCISs are characterized by irregular margins, a non-uniform internal echo texture, and an anteroposterior diameter/width ratio of $\geq 0.7$; however, they were all palpable lesions, $35 \%$ of which had calcifications, and thus, the findings of this study deviates from our results. On the other hand, recent reports on highresolution US make it likely that DCISs that we classified as cystic and solid lesions in this study correspond to the solid and cystic lesions comprising single or multiple hypoechoic masses described by Moon et al. [6] and to "Apart from the small size of the nonpalpable and mammographically occult lesions: complex cysts" described by Wolfgang [1]. However, these reports also deal with non-palpable cystic or solid masses and DCISs with shapes similar to that of benign lesions, and it is not mentioned that $79 \%$ of findings accounted for masses detected by US alone. US findings of DCISs manifesting solid and cystic masses are characterized by those observed in benign diseases, and it is difficult to differentiate DCIS detected by US alone from benign diseases $[1,2,6]$. In the present study, there were many cases in which it was difficult to differentiate DCISs from benign lesions based on shape, margins, etc., and it appeared necessary to conduct detailed investigation mainly via aspiration biopsy cytology.

In this study, mass diameters of the echo group were half that of the MMG/PE group and consistent with those in other reports [1, 17, 18]. Furthermore, localized lesions with few extensive intraductal components were frequently observed, and the Van Nuys classification [8] tumor grades of the echo group were significantly low. In the echo group, all cases with lesions of $<5 \mathrm{~mm}$ were Van Nuys classification grade 1 . Tumor grades were also significantly low compared to those of lesions $\geq 5 \mathrm{~mm}$ of the MMG/PE group. In addition, comparison of cases with lesions $\geq 5 \mathrm{~mm}$ in both groups showed that tumor grades of the echo group were lower than those of the MMG/PE group.

This suggested that cases with lesions of $<5 \mathrm{~mm}$ detected by US alone can be successfully treated by local resection $[19,20]$. The prospective study by ultrasound findings will be necessary in the future. Determination of the course of treatment of such lesions is a subject of future investigation.

DCISs detected by US alone were of low tumor grades when classified by Van Nuys classification and were characterized by frequent localized lesions with few extensive intraductal components, suggesting the possibility of successful treatment by local resection. 


\section{References}

1. Buchberger W, Dekoekkoek-Doll P, Springer P, Obrist P, Dunser $M$. Incidental findings on sonography of the breast:clinical significance and diagnostic workup. Am Roentgenol. 1999;173:921-7.

2. Buchberger W, Niehoff A, Obrist P, DeKoekkoek-Doll P, Dunser M. Clinically and mammographically occult breast lesions:Detection and classification with high-resolution sonography. Semin Ultrasound CT MR. 2000;21:325-36.

3. Gordon PB, Goldenberg SL. Malignant breast masses detected only by ultrasound. Cancer. 1995;76:626-30.

4. Schoonjans JM. Sonographic appearance of ductal carcinoma in situ diagnosed with ultasonographycaly guided large core needle biopsy. J Ultrasound Med. 2000;19:449-57.

5. Sickles EA. Sonographic detectability of breast calcifications. Proc SPIE. 1983;419:51-2.

6. Moon WK, Myung JS. US of ductal carcinoma in situ. RadioGraphics. 2002;22:269-80.

7. Koji T, Ayumi I, Naomi Y. Oue approach for breast cancer screening using both mammography and echoreaphy, with special reference to detection of nonpalpable minute invasive cancer.(in Japanese with English abstract) J Jpn Assoc Breast Cancer Screen. 2007;16:60-5.

8. Melvin JS, David NP, James RW, William J, et al. Prognistic classification of ductal carcinoma-in situ. LANCET. 1995;345:1154-7.

9. Frykberg ER. An overview of the history and epidemiology of ductal carcinoma in situ of the breast. Breast J. 1997;3:227.
10. Schnitt SJ, Silen W, Sadowsky NL, Connolly JL. Ductal carcinoma in situ (intraductal carcinoma) of the breast. N Engl J. 1988;318:898-903.

11. Dershaw DD. Ductal carcinoma in situ: mammographic finings and clinical implications. Radiology. 1989;170:411-5.

12. Stomper PC. Clinically occult ductal carcinoma in situ detected with mammography: analysis of 100 case with radiologic-pathologic correlation. Radiology. 1989;172:235-41.

13. Ikeda DM, Anderson I. Ductal carcinoma in situ: atypical mammographic appearances. Radiology. 1989;172:661-6.

14. Orel SG, Reynolds C, Schnall MD, Solin LJ, Sullivan DC. MR imaging of ductal carcinoma in situ. Radiology. 1997;202:413-20.

15. Holland R, Peterse JL, Millis PR. Ductal carcinoma in situ: a proposal for a new classification. Semin Diagn Pathol. 1994;11:167-80.

16. chen S-C, cheung Y-C. Sonographic differentiation of invasive and intraductal carcinomas of the breast. Br J Radiol. 2003;76:600-4.

17. Leconte I, Feger C, Galant C, Berliere M. Mammography and subsequent whole-breast sonography of nonpalpable breast cancers: the importance of radiologic breast density. AJR. 2003;180:1675-9.

18. Thomas M, Jacob 1, Jeffrey H. Occult cancer in women with dense breast:detection with screening US-diagnostic yield and tumor characteristics. Radiology. 1998;207:191-9.

19. Silverstein MJ, Lagios MD, Craig PH. A prognostic index for ductal carcinoma in situ of the breast. Cancer. 1996;77:2267-74.

20. Lagios MD, Margolin FR, Rose MR. Mammographically detected ductal carcinoma in situ. Cancer. 1989;63:618-24. 